\title{
Teaching Skin Cancer Detection to Medical Students Using a Dermoscopic Algorithm
}

Peggy R. Cyr, MD, MA | Wendy Craig, PhD | Hadjh Ahrns, MD | Kathryn Stevens, FNP | Caroline Wight | Elizabeth Seiverling, MD

PRiMER. 2021;5:6.

Published: 2/8/2021 | DOI: 10.22454/PRiMER.2021.304379

\section{Abstract}

Introduction: Early detection of melanoma skin cancer improves survival rates. Training family physicians in dermoscopy with the triage amalgamated dermoscopic algorithm (TADA) has high sensitivity and specificity for identifying malignant skin neoplasms. In this study we evaluated the effectiveness of TADA training among medical students, compared with practicing clinicians.

Methods: We incorporated the TADA framework into 90-minute workshops that taught dermoscopy to family physicians, primary care residents, and first- and second-year medical students. The workshop reviewed the clinical and dermoscopic features of benign and malignant skin lesions and included a hands-on interactive session using a dermatoscope. All participants took a 30-image pretest and a different 30-image posttest.

Results: Forty-six attending physicians, 25 residents, and 48 medical students participated in the workshop. Mean pretest scores were $20.1,20.3$, and 15.8 for attending physicians, resident physicians and students, respectively $(P<.001)$; mean posttest scores were $24.5,25.9$, and 24.1 , respectively $(P=.11)$. Pre/posttest score differences were significant $(P<.001)$ for all groups. The medical students showed the most gain in their pretest and posttest scores.

Conclusion: After short dermoscopy workshop, medical students perform as well as trained physicians in identifying images of malignant skin lesions. Dermoscopy training may be a valuable addition to the medical school curriculum as this skill can be used by primary care physicians as well as multiple specialists including dermatologists, gynecologists, otolaryngologists, plastic surgeons, and ophthalmologists, who often encounter patients with concerning skin lesions.

\section{Introduction}

Skin cancer affects 1 in 5 Americans. ${ }^{1}$ Early detection of melanoma improves survival. ${ }^{1}$ Dermoscopy is a noninvasive imaging technique using a handheld dermatoscope. Dermoscopy allows for visualization of subsurface structures. In trained hands, dermoscopy is known to improve diagnostic accuracy of physicians in the detection of skin cancer. ${ }^{2,3}$

Prior studies have shown that medical students who receive a dermoscopy tutorial are better at diagnosing 
skin lesions compared with students not receiving the dermoscopy intervention. ${ }^{4,5}$ Furthermore, Chen et al showed that medical students receiving skin cancer training with dermoscopy showed sustained and even improved performance on a 10 -image test 1 year later. ${ }^{6}$ Others have used smartphone wallpapers and video dermoscopy to train medical students, both showing a positive effect. ${ }^{7,8}$

TADA was created to simplify the dermoscopic evaluation of skin growths. TADA workshops can be given in 90 minutes. Physicians participating in the workshops show improved sensitivity and specificity for diagnosing benign and malignant skin growths. ${ }^{9,10}$ This study sought to compare student data from TADA training with that of practicing primary care clinicians.

\section{Methods}

During 2019, we invited all primary care attending physicians and trainees affiliated with our institution to attend one of a series of dermoscopy training workshops. Similarly, we invited all first- and second-year medical students at Tufts University School of Medicine (TUSM) to attend a dermoscopy training workshop on their Boston campus. The Institutional Review Boards at both MaineHeath (physicians) and TUSM (medical students) reviewed and approved the study.

Each workshop lasted 90 minutes and the material presented was the same at every level of provider training. All participants took a 30 -image pretest and a 30-image posttest of different images. Participants were asked to classify the image as melanocytic nevus, dermatofibroma, seborrheic keratosis/lentigo, angioma, or skin cancer. Figure 1 shows a schematic of the methods.

After the pretest, clinical and dermoscopic images of common benign skin growths (dermatofibroma, angioma, and seborrheic keratosis) were reviewed. Dermoscopic features of skin cancer (using TADA) were also reviewed. None of the test images were used in the presentation. A portion of the workshop included hands-on practice with dermatoscopes.

\section{Results}

We summarized all categorical demographic and Likert scale data as frequencies (n, \%). For each participant, we summarized pre- and posttest data as the proportion of correct answers out of 30 questions. We then compared the distributions of these proportions between pre- and post-TADA tests by $t$ test and among participants at different training levels by analysis of variance (ANOVA). We performed all analyses using SPSS Statistical Software, version 25 (IBM SPSS Inc., Armonk, NY).

Table 1 shows demographic and practice characteristics for the 148 providers and students who participated in TADA dermoscopy training. The majority were female (67.6\%), aged less than 40 years (70.3\%), and with the exception of medical students, all practiced in a primary care specialty. The 100 providers varied in their experience with evaluating skin lesions; $56 / 97$ (57.7\%) routinely performed skin biopsies, but only 10/98 (10.2\%) reported prior formal training in dermoscopy (Table 2). Among the 16/97 (16.6\%) of providers who reported that they currently use dermoscopy in practice, $50 \%$ used the technique once per month or less, and $6 / 16(37.5 \%)$ reported lack of confidence in using the technique.

Table 3 shows scores for the participants who completed the pre-TADA $(n=139)$ and post-TADA $(n=134)$ image identification tests. Where numbers allowed, we compared pre- and posttest scores by provider type; mean scores increased significantly $(P<.001)$ after training for attending physicians $(20.5 \pm 2.5$ vs $24.5 \pm 2.6)$, resident physicians $(20.3 \pm 3.3$ vs $25.9 \pm 1.8)$, nurse practitioners ( $17.8 \pm 3.1$ vs $23.6 \pm 3.4)$, and medical students $(15.8 \pm 3.8$ vs 23.6 \pm 3.4$)$. Prior to the TADA training there was a significant difference in test scores among providers with different training types $(P<.001)$, with highest scores among the most experienced providers (attending 
physicians) and lowest scores among the least experienced participants (medical students). These differences were not evident in post-TADA testing $(P=.11)$. In all provider groups there was a significant $(P<.001)$ pre/post TADA increase in the proportion of skin cancer.

Figure 1 shows the pre/post differences in test score were significantly different among the provider types $(P<.001$ by ANOVA). The greatest gain in improvement of test scores was by the medical students. In pairwise post hoc testing, with Bonferroni's correction for multiple comparisons, the differences in test score were significantly different between medical students and attendings $(P<.001)$ and between medical students and residents $(P=.021)$, but not between residents and attendings $(P=.67)$.

\section{Conclusions}

Skin cancer is common. Primary care physicians are often the first medical practitioner approached when a patient has a growth of concern. Dermoscopy has been shown to aid in the detection of skin cancer and distinguishing between benign and malignant skin growths. Many practicing clinicians desire additional dermatology and dermoscopy training. Introducing the use of dermoscopy in medical school might stimulate interest in learning dermatology and be beneficial for those going into primary care as well students pursuing specialties such as gynecology, otolaryngology, plastic surgery, and ophthalmology. ${ }^{11}$ Based on our results, dermoscopy training need not be elaborate. A 90-minute interactive workshop using TADA allowed students to accurately identify benign and malignant dermoscopic images. The ability of students and practicing clinicians to equally acquire new knowledge using TADA is particularly desirable given many institutions do not currently have dermoscopy champions to teach medical students. TADA training can be done via e-learning or in a workshop setting. E-learning would allow novices of all levels to embark on training together. ${ }^{12}$ In our study all provider types (attending physicians, medical residents, nurse practitioners, and medical students) showed significant improvement in detecting skin cancer following TADA training. The medical students may have shown the greatest gain in their scores as they are the most accustomed to learning large amounts of new information. The medical students achieved similar scores to attending physicians despite their lower baseline scores.

Our study shows that the introduction of dermoscopy training in the first and second year of medical school results in the ability of medical students to accurately identify dermoscopic images of skin cancer and benign skin growths at a rate that is comparable to more seasoned clinicians. Introducing dermoscopy in the medical school curriculum might aid early skin cancer detection regardless of the medical students' future career choice.

Our study has several limitations. The study had a small sample size and only one medical school was involved. The students involved in the study were volunteers, so this may be biased towards students more interested in care of the skin. The study also involved only medical students in the first of second year of their training. There is concern that medical students' knowledge could atrophy over time, although others have shown sustained and improved scores after a 1 year on a 10 -image test. ${ }^{6}$ To address this concern, medical students will now have opportunities for additional dermoscopy training during their second-year dermatology block. In addition, students will be invited to participate in monthly dermatology training sessions as part of their primary care third-year clerkships. These additions will provide spaced review that is important for longterm memory.

\section{Tables and Figures}


Figure 1: Difference in Test Scores Before and After Workshop, Stratified by Provider Training

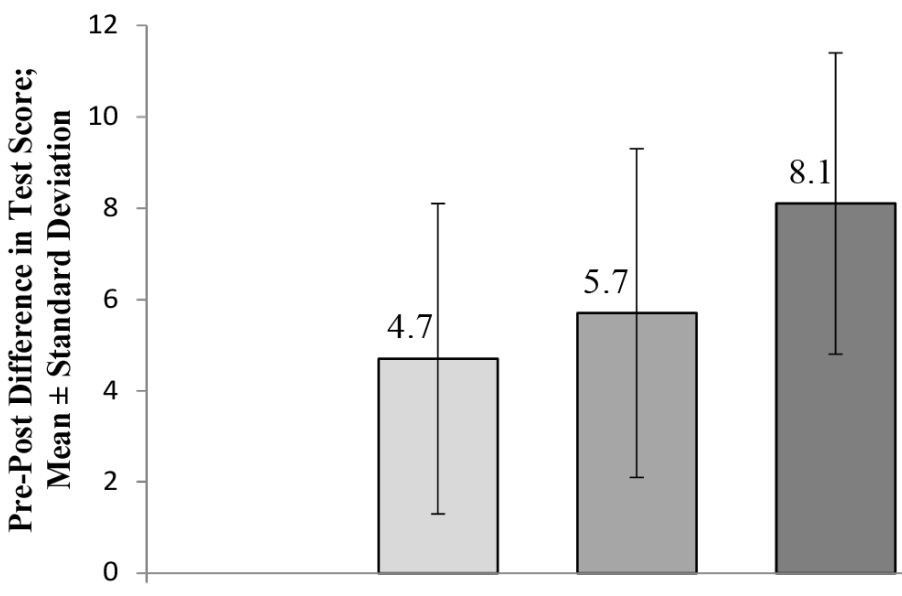

\author{
$\square$ Attendings \\ $\square$ Residents \\ $\square$ Medical students
}

Paired pre/posttest score data were available for 46 medical students, 42 attendings, and 23 residents. Pre/post differences in test score were significantly different among the provider types $(P<.001$ by analysis of variance). In pairwise post hoc testing, with Bonferroni's correction for multiple comparisons, the differences in test score were significantly different between medical students and attendings $(P<.001)$ and between medical students and residents $(P=.021)$, but not between residents and attendings $(P=.67)$.

Table 1: Demographic and Training Characteristics of Dermoscopy Trainees $(\mathrm{N}=148)$

\begin{tabular}{|l|c|}
\hline \multicolumn{1}{|c|}{ Characteristic Age Group (Years) } & Frequency, $\mathbf{n}(\%)$ \\
\hline Female sex & $100 / 146(68.5)$ \\
\hline \multicolumn{1}{|c|}{ Araining Type ${ }^{1}$} & $67 / 146(45.9)$ \\
\hline $31-40$ & $37 / 146(25.3)$ \\
\hline $41-50$ & $18 / 146(12.3)$ \\
\hline $51-60$ & $17 / 146(11.6)$ \\
\hline $61-70$ & $6 / 146(4.1)$ \\
\hline $71-80$ & $1 / 146(0.7)$ \\
\hline & \\
\hline Attending & $51(34.4)$ \\
\hline Resident & $26(17.6)$ \\
\hline Fellow & $1(0.7)$ \\
\hline Nurse practitioner & $18(12.2)$ \\
\hline Physician assistant & $3(2.0)$ \\
\hline Medical student & $48(32.4)$ \\
\hline Other/unspecified & $1(0.7)$ \\
\hline & \\
\hline Family medicine & $62(62.0)$ \\
\hline Internal medicine & $27(27.0)$ \\
\hline Medicine/pediatrics & $7(7.0)$ \\
\hline Other & $3(3.0)$ \\
\hline
\end{tabular}

1 Includes $n=32$ in year $1, n=15$ in year 2 , and $n=1$ with training year not provided.

2 Excludes 48 medical students and 1 provider with other/unspecified training type. 
Table 2: Dermatology-Related Practices Among 100 Trained Providers

\begin{tabular}{|c|c|}
\hline Variable & Frequency, $\mathbf{n} / \mathbf{n}(\%)$ or median [range] \\
\hline Years evaluating skin lesions ( $\mathrm{n=92}$ ) & $7.5[0-40]$ \\
\hline Formal training in dermoscopy & 10/98 (10.2) \\
\hline Access to dermoscopy in clinic & $64 / 97(66.0)$ \\
\hline Current use of dermatoscope & $16 / 97(16.5)$ \\
\hline \multicolumn{2}{|l|}{ Frequency of dermoscopy use } \\
\hline Daily & $2 / 16(12.5)$ \\
\hline $2-3$ x/week & $1 / 16(6.2)$ \\
\hline $1 /$ week & $2 / 16(12.5)$ \\
\hline $2-3 \times /$ month & $3 / 16(18.8)$ \\
\hline $1 /$ month & $4 / 16(25.0)$ \\
\hline$<1 /$ month & $4 / 16(25.0)$ \\
\hline \multicolumn{2}{|c|}{ Confidence in dermoscopy to evaluate a skin growth } \\
\hline 1 (not confident) & $6 / 16(37.5)$ \\
\hline 2 & $2 / 16(12.5)$ \\
\hline 3 & $6 / 16(37.5)$ \\
\hline 4 (very confident) & $2 / 16(12.5)$ \\
\hline Currently perform skin biopsies & $56 / 97(57.7)$ \\
\hline Shave biopsy & $49 / 100(49.0)$ \\
\hline Punch biopsy & $49 / 100(49.0)$ \\
\hline Excisional & $37 / 100(37.0)$ \\
\hline Years performing skin biopsies $(n=46)$ & $7.5[0-27]$ \\
\hline \multicolumn{2}{|l|}{ Reason(s) for not performing skin biopsies } \\
\hline Inadequate supplies to do procedure & $9 / 100(9.0)$ \\
\hline Inadequate staffing to do procedure & $4 / 100(4.0)$ \\
\hline Lack of procedural training & $32 / 100(32.0)$ \\
\hline Lack of time in clinic schedule & $21 / 100(21.0)$ \\
\hline Uncertain how to manage biopsy results & $3(3.0)$ \\
\hline
\end{tabular}


Table 3: Pre/Postdermoscopy Test Scores, Stratified by Provider Type

\begin{tabular}{|c|c|c|c|c|c|}
\hline \multirow{2}{*}{ Provider Type } & \multicolumn{2}{|c|}{ Pretest Score ${ }^{1}$} & \multicolumn{2}{|c|}{ Posttest Score' } & \multirow{2}{*}{$\begin{array}{c}\text { P Value (Prel } \\
\text { Post) })^{2}\end{array}$} \\
\hline & $\mathrm{n}$ & Mean (SD) & n & Mean (SD) & \\
\hline Attending & 45 & $20.1(2.5)$ & 46 & $24.5(2.6)$ & $<.001$ \\
\hline Resident & 25 & $20.3(3.3)$ & 23 & $25.9(1.8)$ & $<.001$ \\
\hline Medical student & 48 & $15.8(3.8)$ & 46 & $24.1(2.8)$ & $<.001$ \\
\hline Nurse practitioner & 17 & $17.8(3.1)$ & 14 & $23.6(3.4)$ & $<.001$ \\
\hline Physician assistant & 2 & $18.5(5.0)$ & 3 & $25.0(1.7)$ & - \\
\hline Fellow & 1 & $24.0(-)$ & 1 & $24.0(-)$ & - \\
\hline Other & 1 & $15.0(-)$ & 1 & $21.0(-)$ & - \\
\hline$P$ value (within test) ${ }^{3}$ & & $<.001$ & & 0.11 & \\
\hline
\end{tabular}

${ }^{1}$ Maximum score for each test was 30 points (1 point/image, 30 images); data are shown as mean (standard deviation).

${ }^{2}$ Independent $t$ test; pre/post difference, by provider type

${ }^{3}$ Analysis of variance, difference among provider types within pretest and posttest

\section{Acknowledgments}

Financial Support: This work was supported in part by the Northern New England Clinical and Translational Research grant U54GM115516.

\section{Corresponding Author}

Peggy R. Cyr, MD, MA

272 Congress Street, Portland, ME 04101. 207-838-8767. Fax: 207-874-1918.

cyrp@mmc.org

\section{Author Affiliations}

Peggy R. Cyr, MD, MA - Department of Family Medicine, Maine Medical Center, Portland, ME I and Tufts University School of Medicine, Boston, MA

Wendy Craig, PhD - Maine Medical Center, Portland, ME

Hadjh Ahrns, MD - Maine Medical Center, Portland, ME

Kathryn Stevens, FNP - Maine Medical Center, Portland, ME

Caroline Wight - Tufts University School of Medicine, Boston, MA

Elizabeth Seiverling, MD - Maine Medical Center, Portland, ME I and Tufts University School of Medicine, Boston, MA

\section{References}

1. American Cancer Society. Cancer Facts and Figures 2020. Atlanta, GA: American Cancer Society; 2020. https://www.cancer.org/content/dam/cancer-org/research/cancer-facts-and-statistics/annual-cancerfacts-and-figures/2020/cancer-facts-and-figures-2020.pdf. Accessed January 22, 2021.

2. Vestergaard ME, Macaskill P, Holt PE, Menzies SW. Dermoscopy compared with naked eye examination for the diagnosis of primary melanoma: a meta-analysis of studies performed in a clinical setting. $\mathrm{Br} \mathrm{J}$ Dermatol. 2008;159(3):669-676. doi:10.1111/j.1365-2133.2008.08713.x

3. Wozniak-Rito A, Zalaudek I, Rudnicka L. Dermoscopy of basal cell carcinoma. Clin Exp Dermatol. 2018;43(3):241-247. doi:10.1111/ced.13387

4. Liebman TN, Goulart JM, Soriano R, et al. Effect of dermoscopy education on the ability of medical 
students to detect skin cancer. Arch Dermatol. 2012;148(9):1016-1022.

doi:10.1001/archdermatol.2012.509

5. Tschandl P, Kittler H, Schmid K, Zalaudek I, Argenziano G. Teaching dermatoscopy of pigmented skin tumours to novices: comparison of analytic vs. heuristic approach. J Eur Acad Dermatol Venereol. 2015;29(6):1198-1204. doi:10.1111/jdv.12790

6. Chen LL, Liebman TN, Soriano RP, Dusza SW, Halpern AC, Marghoob AA. One-year follow-up of dermoscopy education on the ability of medical students to detect skin cancer. Dermatology. 2013;226(3):267-273. doi:10.1159/000350571

7. Cho HG, Sheu SL, Chiang A, Nord KM. Standard dermoscopy and videodermoscopy as tools for medical student dermatologic education. Dermatol Pract Concept. 2018;8(1):39-42. doi:10.5826/dpc.0801a08

8. Wang FY, Liu RF, Chuang G, Yang CH, Chang YY. Smartphone wallpapers for dermoscopy training in medical students and residents. Int J Dermatol. 2019;58(3):345-349. doi:10.1111/ijd.14338

9. Seiverling EV, Ahrns HT, Greene A, et al. Teaching benign skin lesions as a strategy to improve the Triage Amalgamated Dermoscopic Algorithm (TADA). J Am Board Fam Med. 2019;32(1):96-102. doi:10.3122/jabfm.2019.01.180049

10. Rogers T, Marino ML, Dusza SW, et al. A Clinical Aid for Detecting Skin Cancer: The Triage Amalgamated Dermoscopic Algorithm (TADA). J Am Board Fam Med. 2016;29(6):694-701. doi:10.3122/jabfm.2016.06.160079

11. Wu X, Marchetti MA, Marghoob AA. Dermoscopy: not just for dermatologists. Melanoma Manag. 2015;2(1):63-73. doi:10.2217/mmt.14.32

12. Susong JR, Ahrns HT, Daugherty A, Marghoob AA, Seiverling EV. Evaluation of a virtual basic dermatology curriculum for dermoscopy using the Triage Amalgamated Dermoscopic Algorithm (TADA) for novice dermoscopists. Journal of the American Academy of Dermatology. 2019;83(2):59-592.

doi:10.1016/j.jaad.2019.05.097

Copyright $@ 2021$ by the Society of Teachers of Family Medicine 